\section{CON1F-8903335- - 146 \\ SLAC-PL'B-9933 \\ March 1989}

(A)

CONSTRUCTION OE SYMPLECTIC TULLTURN MAPS BY APPLICATION OF AN ARBITRARY TRACKING CODE'

\author{
ROBERT \&. WARNOCK \\ Stanfond Linear Accelerator Center \\ Stanford University, Stanford, Californis g4309
}

\title{
SLAC-PUB- -4933
}

\section{ABSTRACT}

A map to describe propagation of particlea through any section of a nonlinear lattice may be represented a a Taylor expansion ahout the origin in phase space. Altbough the technique to compute the Thylor coefficients has been improved recently, the expansion may fail to provide adequate accuracy in regions where nonlinear efiects are substactial. A representation of the map in asgle-action coordipates, with the angle dependence given by a Fourier series, and the action dependence by polynonials in $J^{1 / 2}$, may be more successful. Maps of this form are easily constructed by taking Fouriet transforme of results from an arbitrary symplectic tracking code. Examples are given of one-turn and two-lurn maps for the SLC North Damping Ring in a strongly nonlinear region. Results for accuracy and speed of evaluation of the maps are quite encouraging. It seems lessible to make accurale maps for the SSC by this method.

\section{INTRODUCTION}

Symplectic maps to describe linear motion are easy to construct and are used every day in analysis and operation of acceleralors. Maps to describe nonlineas motion are more difficult to manage. Aithough nonlinear maps beve been used success. fully for certajn purposes, mainly to represent the approximate effect of one or a lew nonlinear elements, 1.2 they have nat become a standard tool of the trade. This situation mey be due to the lack of a simple method to construct maps of adequate accuracy and scope. The present paper describes an extremely easy procedure for construeting acrurate maps with the belp of any tracking code.

For the study of circular machines, maps describiag one full turn are of special interest. (More generally, th-turn maps could be interesting, $\mathbf{n}$ bejing a small inleger or a rational number). A full-turn map could provide economical long-term tracking. provided that one evaluation of the map takes leas time than element-by-element tracking through one tum. This sbould happen for sufficiently large lattices, wince complexity of the map (the number of terms tequired to reptesent in in come expansion) does not, in general, increane with the length of the lattice. Another application of full-turn maps (of great importance in my view; occurs in a scheme to study invariant surfaces in phase space and to derive long-term bounds on the motion." One can find an equation for detertnination of the inverient surface that refers only to the mop, making no reference to the underlying Hamiltonian. This formulation avoids the multiple integrations through the lattice that are aeeded to solve the same problem by the Hamilton-Jacobi method."

Since the ultimate goal is to study the dynamic aperture. a really useful map should be cceurate in regions of large am. plitude and strong nonlinearity. In wuch regions an ansilysis of stability is specially sensitive to errors, of that one should be scrupulous in maximizing accuracy of the map for a given expenditure of computational resource.

$A$ general $M$ talkes + phase space point $\approx$ at orbital location s to a point $z^{\prime}$ a orbita location o":

$$
\mathbf{M}\left(z ; s, s^{\prime}\right)=z^{\prime} \text {. }
$$

The vsual practice has been to represent $M$ a a power series in the components of $\mathbf{2}$ a Taylor expansion about the origin in phase space. Recently Betz ba introduced a technique Which allows one to compute Taylor coefficients of muth higher order than wa possible previously. In spite of this advance

- Mork supported by the Department of Energy. contract DE-AC03-76SF0051.5.

\section{DE89 012270}

in technique, I believe that the Taylor series is not the appro priate tool for representing the map at large amplitude. Rather than using only the values of the mop and its derivatives at $z=$ 0 , and making a long extrapalation, one should use values of the map in the actual large-amplitude region of interest. Those val. ues are readily determined by any tracking code. The problem of constructiog a formula for the map is then a problem of inter. polation of values at points close to the orbits of interest, rather than extrapolation from foints far away. It is un/lul to vieu the problem in angte-action coordinat s. For transterse motion in two degres of freedom the angle $=\left(\phi_{1}, \phi_{2}\right)$ and action $I=\left(I_{1}, I_{2}\right)$ are related to Carlesian ph. - space coordinates $x=$ $\left(x_{1}, I_{2}\right), p=\left(p_{1}, p_{2}\right)$ through the lattice whetions $p_{1}$ as follows:

$$
\begin{gathered}
x_{1}=\left(2 f_{2} \theta_{1}(s)\right]^{1 / 2} \cos \theta_{1} . \\
\left.p_{2}=s_{1}^{\prime}=-\left[2 f_{1} / \theta_{1}(s)\right]^{1 / 2}\left[\sin \phi_{1}-\frac{1}{2} F_{.}, 1\right) \cos \phi_{1}\right] .
\end{gathered}
$$

Primes denote derivatives with respect to arc length $s$ on the reference orbit.

On a typieal orbit, the action I has relatively little varia tion (for linear motion, none at all), while the angle corers the full intcrel $(0,2 \pi)$. Consequently, in cons: ucting a map by interpolation, ope should expect to need lewe mesh points in I than in $\$$, provided that the map is intende to be aceurate only in a band in action space,

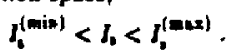

By restricting the map to such a band, and usics, several maps if necessary to cover different bands, one gains in accurary and in speed of evaluation of the map. Note that such a strategy is nol postible in the appranch using Taylor series.

\section{REPRESENTATION OF THE MAP}

The map to describe evolution of orbits from location $s$ to location $s^{\prime}$ will be written as

$$
\begin{aligned}
& \gamma^{\prime}=\boldsymbol{C}+\mathbf{A}\left(1,9 ; 3, s^{\prime}\right) \\
& \Gamma=I+B\left(I, 9 ; s, s^{\prime}\right)
\end{aligned}
$$

Henceforth the arguments $s, s^{r}$ are suppressed, since fixed volues such as $s=0, s^{\prime}=C$ are contemplated, where $C$ is the circumference of the refereace orbit. In accord with the definition of 5 , the functions A,B must be $2 \pi$-periodic in each $\phi_{\text {. }}$ It is therefore natural to represent them by Fourier series. As in the computation of invariant surface, one finds that a sparse set of Fourier modes is dominant. For a given accuracy, mode numbars up to some maximum bave to be included. but for many mode numben below that maximum the Fouriet coefficients are negligible. Thus, it is efticient to represent $A$ and $B$ in a form such as

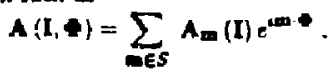

where the eer $S$ includes all $m$ for which $\left|A_{m}\right|$ is geater than some $c$ times the larget $\left|\boldsymbol{A}_{\mathrm{m}}\right|$.

To calculate the Fourier coefficients $A_{\text {mo }}(I)$ (say for a oneturn map), the function $-.-A=A(1,+)$ is evaluated at fixel I on a uniform mesh in 6 .

$$
\phi_{1}=\frac{2 \times n}{N}, \quad n=0.1, \ldots, N-1 .
$$

The evaluation is done by rumbing a tracking code for one luth, using Egs. (1.2) and (1.3) to Iranslate from Cartesing to. 
action-angle variables. The values on the mesh provide the data for a Fast Fourier Transform, which yields the coefficients $\mathbf{A}_{\mathbf{m}}$. A small technical problem arises because $Y$ is computed nodulo $2 \pi$, which means that when is varied, either component of $\Phi$ can reach $2 \pi$ and then suddenly jump to zero. Of course, one does not wish to take the Fourier transform of a disecintinuous function. Value of an equivalent fupction without jumps must fipt be constructed. That is easy to dn: if $\phi_{i}^{\prime}$ drops by a large fraction of $2 \pi$ (say $80 \%$ of $2 \pi$ ) when $\phi$, is moved from one point to the next on the mesh (2.4), then $\phi^{\prime}$, is incremented by $2 \pi$.

Hepeating the calculation for many values of $I$, one finds that the Fourier coefficients are smooth functions of I without much structure, usually monotonic, over the region encountered on a 15 pical orbit. I have experimented with two ways to represent these fuactions: (a) interpolation by polynomials in the variables $\epsilon_{1}=I_{1}^{1 / 2}$, and (b) spline interpolation in the same variables. In examples studied to date, choices (a) and (b) usualjy gave similar results, atthough (a) worked considerably bettcr in the example reported below. The programming required for $(a)$ is extremely simple. In one dimension, the polyncmial approximation of the Fourier coefficient is given in Lagrange form as

$$
A_{m}(I)=\sum_{m=1}^{n} A_{m}\left(I_{s}\right) \lambda_{0}(\xi),
$$

whice the Lagrange factors are

$$
\lambda_{\lrcorner}(\xi)=\prod_{t \neq \pm} \frac{\xi-\xi_{t}}{\xi_{m}-\xi_{t}} .
$$

Thus. the approximating polynomial passes through the correct values $A_{m}\left(I_{0}\right)$ at the mesh points $\xi_{0}=I_{0}^{1 / 2}$. According to the work of numerical analysts, ${ }^{5}$ one should avoid a uniform destribution of mesh points, which can give surprisingly bad results in the limit of large $n$. Following the discussion of de Boor. ${ }^{6}$ I have used the expanded Chebyshev puints, narrely.

$$
\begin{gathered}
\xi_{n}=\frac{1}{2}\left[\xi_{n}+\xi_{n}+\left(\xi_{1}-\xi_{n}\right) \frac{\cos \left[\frac{\pi}{2 n}(2 s-1)\right]}{\cos \left[\frac{\pi}{1 n}\right]}\right] . \\
s=1,2, \ldots, n .
\end{gathered}
$$

In two dimensions rith polynomial interpolation the complete expression for the component of the map is

$$
\Phi^{\prime}=++\sum_{m \in S}\left[\sum_{j=1}^{m_{j}} \sum_{t=1}^{m_{2}} A_{m}\left(I_{1} \ldots I_{2 t}\right) \lambda_{t}^{(1)}\left(\xi_{1}\right) \lambda_{t}^{(2)}\left(\xi_{2}\right)\right] e^{(m+*} \text {, }
$$

where $\lambda_{a}^{(1)}$ is the Lagrange factor for mesh points $\xi_{0}^{(0)}$. Thus, the data that fully characterize ihe map are the complex coeff. cients $\boldsymbol{A}_{\mathbf{m}}, \mathbf{B}_{\mathbf{m}}$ at action values corresponding to the Chebyshev points. The set $S$ of included modes is actually different for each of the four components of the map, usually being smaller for the $\left(t_{1}, t_{2}\right)$ componeats than for $\left(\phi_{1}, \phi_{2}\right)$.

\section{EXAMPLE: SLC NORTH DAMPING RING}

As an example, maps for the SLC North Damping Ring sere constructed. The maps cover a resion of phuse space of this machine that was explored in an accompanying paper. The interpolation polynomials are sixth degroe in each variable $\xi_{1}$ with minimum apd maximum interpolation points at the ends of the intervals

$$
1.5 \times 10^{-6} \leq I_{1} \leq 3.25 \times 10^{-6}, \quad 10^{-6} \leq I_{2} \leq 2.5 \times 10^{-6}
$$

where actions are expressed in meters. The Fourier modes are chosen from an initial set in which $\left|m_{1}\right|,\left|m_{2}\right| \leq I 1$. For each of the four components of the map, all modes with coefficients greater than $10^{-7}$ of the largest coefficient are retained, this selection being made at the maximum values of the actions. For a one-turn map this yields a total of 223 coefficients to describe all four components of the map: for a two-turn map there are 246. The initial, unselected set had 972 independent coefficients; (not all Fourier coefficients are independent, due to reality conditions).

The tracking code used to compute the map coefficients was based on Ruth's fourth-order symplectic integrator. "Tlit code was run with one fourth-order integration step per sextupole: magnet. A short test with two steps per magnet produced map coeficients that wete the same to seven or eight digits. Of course, the code produces a symplectic time evolution. modulo round-off error, whatever the number of integration steps.

To check accuracy of the $n$-th iterate of the map. its value: was compared to the corresponding value obtained from the tracking code. The discrepancy $\delta(n)$ letween the map and tracking at the $n$-th turn is defined as

$$
\delta(n)=\frac{1}{4} \sum_{i=1}^{2}\left[\left|\frac{\phi_{1}-\phi_{1}^{l}}{\phi_{1}}\right|+\left|\frac{l_{1}-\Gamma_{1}}{l_{1}}\right|\right]_{n \cdot t h \text { turn }}
$$

The coordinates from tracking have superseript $t$. those from the map do not.

Table 1 shows values of $\delta(n)$ for one-turn and two-turn maps, for an orbit with initial conditions $\phi_{1}=\phi_{2}=0, l_{1}=$ $I_{2}=2 \times 10^{-8}$ ra. This orbit lies on an invariant surface that is displayed graphicalty in Figs. 1 and 2 of Ref. 7. Since the surface is far from being planar, the motion is highly nonlineas. The artions cover most of the iatervals (3.1). For the one-turn map the discrepancy at one turn was $\delta(1)=5.3 \times 10^{-6}$, whereas for the two-tira map the discrepancy at two turns (i.e., at the first iteration of the map) was $\delta(2)=2.9 \times 10^{-B}$. Table 1 gives the dis. crepancies at $10^{\prime}$ turns, with $p=1, \ldots, 4$. It is remarkable that the discrepancy is still quite small at 10000 turns. It is likely that the orbit generated by the map stays clnse to the corteet invariant surface for many turns beyond ioowo, since it is usual for phase error to build up faster than amplitude error. That is, the orhit generated by the map might lie close to the surface. with out having the correct angular location at a particular turn

Table 1: Discrepancy $\delta$ between Map and Tracking Code

\begin{tabular}{|c|c|c|}
\hline $\begin{array}{c}n=\text { Number } \\
\text { of Turns }\end{array}$ & $\begin{array}{c}\delta(n) \\
\text { One-Turn Map }\end{array}$ & $\begin{array}{c}\delta(n) \\
\text { Two-Turn Map }\end{array}$ \\
\hline 10 & $2.2 \times 10^{-7}$ & $6.8 \times 10^{-8}$ \\
100 & $1.6 \times 10^{-7}$ & $1.3 \times 10^{-6}$ \\
1000 & $7.9 \times 10^{-6}$ & $9.9 \times 10^{-8}$ \\
10000 & $5.8 \times 10^{-1}$ & $6.2 \times 10^{-1}$ \\
\hline
\end{tabular}

\section{SYMPLECTIC CONDITION}

Evidently the maps of this example satisfy the symplectic condition to high accuracy, since they agre very well with the underlyjing gymplectic tracking program. As will be explained presently, it is also possible to create a map that is exactly sym. plectic. One hears the opinion that some loss in accurary can be afforded provided that symplecticity is exact. In particu. lar, a symplectic map might give some phase error while still producing almos the correct structures in phase space (invari. ant tori, resonance islands, chaotic regions). Hard evidence for this premise may be scarce. To check the idea, one could calculate the distance between the orbit of a proposed symplectic map and an accurate, previous) y extablished invariant torus. Phase error would be innocuoys in this iest. The insariant torus can be obtained from tracking data by the fitting procedure of Ref. 7.

To make the map exactly symplectic, it has to be defined implicitly in terms of a generating function $G\left(1, w^{\prime} ; s^{\prime}\right)$ which satisfies the initial condition $G\left(I, S^{\prime} ; s, s\right)=0$. With partial 
derivatives denoted by subacripts. the map $(\Gamma, I) \rightarrow\left(\Gamma^{\prime}, \Gamma\right)$ is defined by the equations

$$
\begin{aligned}
& s=s^{\prime}+G_{1}\left(I, s^{\prime} ; s, s^{\prime}\right) . \\
& I^{\prime}=I+G_{*}\left(I, s^{\prime} ; s, s^{\prime}\right) .
\end{aligned}
$$

To evaluate the map, one has to solve the nonliuear equa. tion (4.1) for 4 , then substitute in (4.2) to obtajn $\mathbf{r}$ as well. This step is not as difficult as it might appear, since one already has a good guess for the solution of (4.1) from the explicit map (2.1) constructed above. It is only nectesary to refine that guess by iteration, say by an application of Newton's method. Such a program was carried out in Rel. 9 in 1 one-dimensional example; one evaluation of the symplectic map took $60 \%$ longer than one evaluation of the explicit but nonsymplectic map.

The generator can be found by numerical integration of the Hamilton.Jacobi equation, as in Ref. 9 . It may also be obtained from the functions $A, B$ already constructed, in such a way that the map induced by the generator is approximately the same as (2 j). (2.2). but of course exactly symplectic. This lat ter deriva. tion of the generator uses a Fourier inversion method similas to that of Ref 9: details will be published elsewhere.

\section{COMPUTATIONAL Cost}

Results concerning compulation time to jterate the map sein farorable, eten though not much has been done to opti. mize computing. On the IBM $30 S$ ! at SLAC the tjme for one it eration of the one-turn map described above is about five times greater than the time to track for one turn with the underlying tracking code. Since the ring bas 72 sextupoles, tracking by a map would so faster than element-by-element tracking in 2 ring with more than $5 \times 72=360$ sextupoles, provided that a map with the same number of terms would suffice. In a ring as big as the Superconducting Super Collider (SSC). a factor of $10000 / 360 \approx 28$ would be gajned by this reckoning. Actually. much bigger gains can be anticipated, since evaluation of the sum (2.8) is a very simple computational problem which undoubtedly can be handled with great efficiency through better programming and hardware. Currently, most of the computing time is absorbed in the sums over $s$ and $t$, which could be speeded up through vector processing.

The ourlook for the cost of constructing mups is perhaps nol quite as favorable, but still quite rearonable. It took the equivalent of $2822 \mathrm{~s}$ turns of element-by-element tracking to construct the ose-curn map described above, and (wice that much fot the two-turn map. Once the map is avilable, howener, it can be used to track economically (Jor hrge rings) from any initial condition within its domain of validity, and, more importantly, be used to study invariant surfaces. Furthermore, one could make maps for several values of momentum, and interpo. - late between them to treat chromatic effects and synchrotron motion. Similarly, tune space could be explored oconomically by interpolating maps for several tunes.

Construction of eccurate maps for the SSC by the present method seems entirely feasibje. The latest symplectic tracking code for the SSC. due to J. Irwin and Y. Yan, would require less than 1.5 min. on the Cray XMP to trake a two dimensiona map like that described above (Fourier modes with $\left|m_{1}\right|,\left|m_{2}\right| \leq 11$; 6th-degree polynomials for action dependence). This figure is for the full SSC lattice, including random multipoles to eightb order and possible magnet misalignments. To include sybchrotron motion, it would probably be sufficient to create maps for 5 to 10 values of momentum. Thus. one ran expert to create maps for the SSC in less than 1.5 minutes of (ray tinn.. that woukd accurately describe betatron motion in strongly won ligeat regions, and also account for synchrotron motion

It is not yet clear as to whether there would be a cosl ad vanlage in using an implicin symplectic map. Since it iakes considerably more time to evaluate the implicit map, it might be wiser to use the explicit map with enough terms to sat isfy the symplectic condition to the desired accuracy.

The question of whether one should work with a onf-lurn map or a multi-turn map deserves continued study. In Table 1. the one-turn and two-turn maps show comparable arcuracy at the $n$ - th turn of tracking. Tracking by the two-turn map goc' almost twice as fast (not exactly twice, because there are a fi'w more Fourier modes), but the map takes twice as long to con. struct. Using the two-turn map. one might be able to study invariant surfares in the neighborhood of a closed ortit of pe riod two. Also, one can imagine a bootstrap opetation in whirh a one-turn map, say, could be used to generale a map for mant turns.

\section{Conclusion}

Maps expressed in angle-action coordinates, with the asiglt dependence given by a Fourier series and the action dependence by polynomials in $j^{1 / 2}$, have a number of adranlages. They ate easy to construct, accurate, and economical to evaluate They should lead to a powerful method to study invariant surfaces and long term stability.

\section{ACKNOWLEDGMENTS}

I thank Johu Irwin and Ronald Ruth for encouraging this work, and Michael Condron for help with programming.

\section{REFERENCES}

1. Nonlinear maps have been used in the computer cade TRAXSPOIT since the early 1960's. See K. L. Brown. D. C. Carey. Ch. Iselin and F. Rothacier, CERS 80-04. 1980; K. L. Brown. R. Belbeoch and P. Bounin. Res. Sri. Instr. 35, 481 (1964).

2. A. J. Dragt et al., Ann. Rev. Nucl. Part. Sci, 38. 455 (1988).

3. R. L. Warnock, R. D. Ruth, W. Gabella and K. Eckiund. SLAC. PLB-4856. Januery 1989.

4. W. E. Gabella. R. D. Ruth and H. L. Marnock, Secand Adranced ICFA Beam Dynamics Wontshop. Lugano. 1988, CERN S8-04: R. L. Warnock and R. D. Ruth. Phys. ica 20D, I (1987).

5. M. Berz, SSC-166, March 1986

6. C. de Boor, "A Proclicai Gude to Splinrs, "Chaps II (Springer, Rew York. j978); T. J. Rjwin. -An Introduc. lion to the Apprortmation of Functuons," (Dover. Xirw York, 1981)

7. R. L. Warnock, R. D. Ruth and K. Ecklund, "Constraction and Fourier Analysis of Invanant Surfaers from Tracking Data," these Proceedings and SLAC-PI'B 4927; see also Ref. 3.

8. R. D. Ruth, unpublished. A similar third-order integrator appears in II D. Ruth. IEEE Trans. Nuc). Sci. 30 (19s:3) 2669.

9. R. L. Warnock. R. D. Ruth and W' Gabelta, SLAC-PL:B 4627 . 


\section{DISCLAIMER}

This repor was prepared as an acoount of work sponsoned by an agency of the United States Government. Neithet the United States Government nor any agency thereof, nor any of their employecs, makes any wattenty, express or implied, of assumes any lezal liability of responsibility for the accurecy, compteleness, or ucefulness of any information, apparates, product, or proces diselosed, of represents that its use would not inftioge privately owned rights Reference herein to any spexific commercial product. proceas, or service by trade name, Irademark, manufacturer, or otherwise does nol necessatly constitute or imply its endorsement, recommendation. or favoring by the Uniled States Government or any agency thereof. The views and opinions of authors expressed herein do not nocessarily state or reflect those of the United States Government of any agency thereof. 